\title{
Inner horizons of fluid contingencies
}

\author{
Experimenting with exotic phenomena using exotic media.
}

S imultaneously comprising multisensory environments, philosophical inquiries and scientific experiments, our installations and performances (www.portablepalace.com) have emerged through unorthodox collaborations with pioneering research groups.

When we initiated our artistic practice about 20 years ago, we established a variety of constraints. To propel our departure from art's age-old infatuation with frozen objects, we precluded the use of solid, fixative and recording media in favour of liquids, gases and plasmas permeated by acoustic vibrations and light emissions. Our conceptual constraints eventually led to the creation of Camera Lucida: Sonochemical Observatory (2003-2008), an artwork exploring the paradoxical phenomenon of sonoluminescence. After vanishing in the impenetrable darkness surrounding the installation, the visitor gradually perceives an undulating cosmos of glowing sound fields. In a spherical chamber filled with 60 litres of water, acoustically imploded air bubbles cyclically shrink to the size of 100 nanometres, at which point they reach temperatures as high as those found on the Sun and emit picosecond flashes of light. There has yet to be a conclusive model accounting for the nearly trillionfold energy amplification underlying sonoluminescence.

We made a leap from collapsing microbubbles to macrobubbles of soap film in 10,000 Peacock Feathers in Foaming Acid (2007). As a multicolour laser beam enters a soap bubble membrane (the chemical

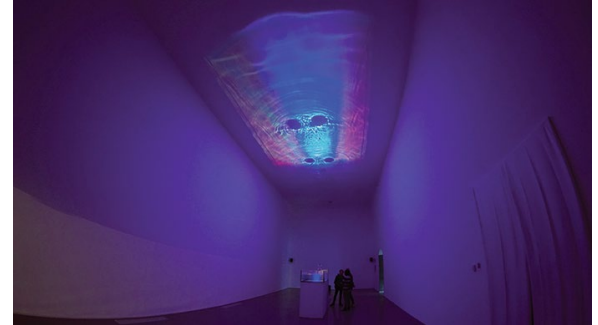

An image from $E R=E P R$ exhibited at Black Hole: Art and Materiality from Informal to Invisible, Galleria d'Arte Moderna e Contemporanea, Bergamo, Italy, 2019.

ancestor of a living cell's lipid membrane), it splits up into a bundle of optical tracks known as polariton-solitons. During the performance, these laser tracks meander through clusters of hemispherical bubbles, which serve as molecule-thin lenses for a $360^{\circ}$ laser projection. Presented in planetaria and other dome-shaped environments, the projection envelopes the audience in an unfathomable magnification of the electrochemical, optical and fluid dynamics within the sensitive skin of a bubble.

In 2016, the primordial foam resurfaced in one of our most challenging endeavours, Luminiferous Drift. Developed in collaboration with Jean-Marc Chomaz of Laboratoire d'Hydrodynamique (École Polytechnique Paris), Hui Lab (University of California, Irvine), Huck Group (Radboud University) and composer Richard Chartier, whose music evokes the environment of a satellite observatory, Luminiferous Drift envisions the climate of a hypothetical planet punctiliously traced by bioluminescent protocells. By means of a microfluidic chip, an enzyme-regulated chemiluminescent solution is injected into a vegetable oil membrane. The resultant double-emulsion protocells are released into a rotating bath of water that recreates Saturn's hexagonal polar vortex.

Another liquid evocation of celestial dynamics, $E R=E P R$ (2017) was a collaboration with the Laser Interferometer Gravitational-Wave Observatory LIGO (Caltech), Jean-Marc Chomaz and composer William Basinski, whose composition used sound files from LIGO detections as source material. Two corotating vortices, joined together by a slender vortical bridge, drift through an enormous aquarium. Shining through the water surface, an expanded laser beam transforms the vortex pair into a moving lens that upwardly projects two black holes encircled by shimmering halos. Vortices may collide with one another (as in the gravitational-wave detections at LIGO), and if the 'wormhole' link between them rips apart, the black holes immediately dissipate. $E R=E P R$ refers to the mindbending conjecture of Juan Maldacena and Leonard Susskind, equating wormholes with quantum entanglement.

\section{Evelina Domnitch ${ }^{\star}$ and Dmitry Gelfand* *e-mail:diarchy@portablepalace.com}

Published online: 7 May 2019 https://doi.org/10.1038/s41565-019-0446-6

\section{The poetry of materials}

With their unique perspectives, artists can become part of the scientific process.

W hen we've worked together we have begun with what we'd consider a shared concept, rather than the exchanging of introspective information. We endeavour towards a rigorous approach to an artistic and scientific practice, resulting in works of scientific art and artistic science. We create objects and explorations that evolve in both directions, and recognize, innately, that the objective is secondary to subjective applications and experiences. This approach has generated a series of what might be generally termed as 'translations', most specifically the rendering of poetic language about a scientific process in that mode, medium or means.

For example, we utilized the selfassembly process of proteins to describe the physical shape of a language or poem through reverse-engineered silk (pictured). By studying the evaporation rate of water alongside the self-assembly process of proteins, we etched a language, made optically visible due to proteins following topographies of the substrate on the nanoscale. The poem itself evokes the scientific and artistic processes. The scientist is indeed an artisan of science.

More recently Thomas has been collaborating with KUKA Robotics on an incredible tool of industrial, scientific and artistic expression that allows the merger of 